\title{
MicroRNA-30e regulates TGF- $\beta$-mediated NADPH oxidase 4-dependent oxidative stress by Snai1 in atherosclerosis
}

\author{
YE CHENG $^{1 *}$, MEILI ZHOU ${ }^{2 *}$ and WENJUN ZHOU ${ }^{3}$ \\ ${ }^{1}$ Department of Cardiology, Xiamen Cardiovascular Hospital Xiamen University, Xiamen, Fujian 361004; \\ ${ }^{2}$ Department of Cardiology, The Second Affiliated Hospital of Xiamen Medical College, Xiamen, \\ Fujian 361021; ${ }^{3}$ Department of Intensive Care Unit, Rui Jin Hospital, Luwan Branch, \\ Shanghai Jiao Tong University School of Medicine, Shanghai 200020, P.R. China
}

Received July 23, 2018; Accepted February 18, 2019

DOI: 10.3892/ijmm.2019.4102

\begin{abstract}
MicroRNAs (miRNAs) are small non-coding RNA molecules that regulate gene expression at a post-transcription level in living organisms. Great attention has been paid to the role of miRNAs in the pathogenesis of atherosclerosis (AS). The present study was designed to investigate the function of miRNA-30e in atherosclerosis and to explore potential mechanisms. The expression of miRNA-30e was decreased in an AS model, compared with the normal group. The downregulation of miRNA-30e increased oxidative stress and reactive oxygen species (ROS) levels in vitro. Then, overexpression of miRNA-30e led to decreased oxidative stress and ROS levels in vitro. The downregulation of miRNA-30e induced the protein expression of Snail, transforming growth factor (TGF)- $\beta$ and mothers against decapentaplegic homolog $2(\operatorname{Smad} 2)$ and suppressed that of NADPH oxidase 4 (Nox4) in vitro. The activation of Snail or TGF- $\beta$ attenuated the effects of miRNA-30e on oxidative stress in vitro. Consistently, the inhibition of Nox4 attenuated the effects of miRNA-30e on oxidative stress in vitro. These findings demonstrated for the first time that miRNA-30e regulated AS by TGF- $\beta$-mediated NADPH oxidase 4-dependent oxidative stress via Snail.
\end{abstract}

\section{Introduction}

Atherosclerosis (AS) is a common disease threatening human health. In recent years, the morbidity of AS is markedly increased in China (1). A previous study demonstrated that

Correspondence to: Dr Ye Cheng, Department of Cardiology, Xiamen Cardiovascular Hospital Xiamen University, 205 Hubin South Road, Xiamen, Fujian 361004, P.R. China

E-mail: chetu960@yeah.net

${ }^{*}$ Contributed equally

Key words: microRNA-30e, atherosclerosis, transforming growth factor- $\beta$, NADPH oxidase 4 , Snail, oxidative stress the inflammatory response serves a key role in the formation process of AS (2). Mononuclear macrophage and lymphocyte, multiple growth factors, cytokines and adhesion molecules are closely correlated with the formation, development and rupture of the atherosclerotic plaque (2). Typically, coronary AS plaque rupture and the induced thrombosis are the important causes leading to acute coronary syndrome and death (3). Furthermore, plaque structure and components also serve an important role in maintaining its stability (1).

MicroRNA (miRNA/miR) is a class of highly conserved non-coding small RNA about 19-25 bp in length, which (4) can bind with target genes (mRNA) through base complementation or partial base complementation. Therefore, it can degrade mRNA or restrain its translation and regulate gene expression at a post-transcription level. miRNA distribution in the human body is tissue specific, which serves a key role in cell proliferation, transformation, migration, metabolism and apoptosis (5).

AS is a complicated multi-factor disease, but its pathogenesis remains unclear (6). It is characterized by destroyed vascular endothelial cell integrity, smooth muscle cell and fibroblast hyperplasia, and lipid atherosclerotic plaque formation under the large and medium-sized endarterium (7). Research indicates that the acute and chronic overloads of reactive oxygen species (ROS) exist in the whole process of AS, which will induce endothelial cell injury and apoptosis. Therefore, ROS serves a key role in the pathogenesis of AS. ROS mainly affect the growth, migration, proliferation and activation of vascular cells (7). Under pathological conditions, ROS are involved in inflammation, endothelial dysfunction, cell proliferation and activation. Recent studies suggest that, diabetes, hypertension, hyperlipidemia, obesity and smoking will induce excessive ROS production $(7,8)$. Furthermore, they will increase lipid peroxidation injury to cells, therefore promoting AS formation and development (8). Transforming growth factor (TGF)- $\beta$ is a multi-functional cytokine, which can regulate cell growth, proliferation, differentiation, migration and apoptosis (9). In addition, it may stimulate the synthesis and secretion of multiple active substances, including cytokines, and inflammatory mediators. Therefore, it participates in the extracellular matrix (ECM) constitution and degradation, and has dual effects of suppression and promotion (10). 
NADPH oxidase (NOX) is demonstrated in research to be a major enzyme involved in ROS production in blood vessels (11). Evidence proves that NOX4 expression in endothelial cells is markedly increased compared with other Nox members (12). Research data also prove that NOX4 serves a key role in endothelial redox reactions and has an important functional regulatory effect on endothelial cell physiology (13). Lai et al (14) demonstrated that miRNA-30e mediated cardioprotection of ACE2 in rats with doxorubicin-induced heart failure. The present study was designed to investigate the function of miRNA-30e in atherosclerosis and to explore potential mechanisms.

\section{Materials and methods}

Atherosclerosis in vivo model. C57BL/6 $(\mathrm{n}=8$; male; 5-6 weeks; $18-20 \mathrm{~g})$ and Apoe $^{-/}(\mathrm{n}=8 ; 5-6$ weeks; $18-20 \mathrm{~g})$ mice were obtained from the Animal center of Xiamen University (Xiamen, China). All mice were housed at $22-23^{\circ} \mathrm{C}$, 55-60\% humidity, $12 \mathrm{~h}$ light and dark cycle, and had free access to water. C57BL/6 mice were the normal control group and were fed normal diet for 12 weeks. The $\mathrm{Apoe}^{-/}$mice were the AS group and were fed the high-fat diet for 12 weeks. Animal experiments were approved by the Animal Care and Utilization Committee of Xiamen Cardiovascular Hospital Xiamen University. Following 12 weeks, mice were injected with $50 \mathrm{mg} / \mathrm{kg}$ pentobarbital sodium and then sacrificed using decollation. The aorta vessel tissue was fixed using $4 \%$ paraformaldehyde for $24 \mathrm{~h}$ at room temperature and made paraffin sections $(5 \mu \mathrm{m})$ for Oil Red O staining at room temperature for $15 \mathrm{~min}$. Tissues were observed using a confocal fluorescent Zeiss Axioplan 2 microscope (magnification, x100; Carl Zeiss MicroImaging; Carl Zeiss AG, Oberkochen, Germany).

Oxidative stress and ROS levels. Malondialdehyde (MDA; cat. no. A003-1), superoxide dismutase (SOD; cat. no. A001-1-1), glutathione (GSH; cat. no. A006-2) and glutathione-peroxidase (GSH-PX; cat. no. A005) levels were measured using ELISA kits from cells or blood vessel tissue (all from Nanjing Jiancheng Bioengineering Institute, Nanjing, China). The ROS level was measured in cells using ELISA kits (cat. no. E004; Nanjing Jiancheng Bioengineering Institute). In addition, ROS level was analyzed using ROS probe and observed using a Zeiss Axioplan 2 (Carl Zeiss MicroImaging; Carl Zeiss AG).

Quantitative polymerase chain reaction $(q P C R)$ and gene microarray hybridization. Total RNA was extracted from cells or blood vessel tissue using TRIzol ${ }^{\circledR}$ reagent (Invitrogen; Thermo Fisher Scientific, Inc., Waltham, MA, USA). Reverse transcription (RT) was performed with the TaqMan miRNA RT kit (Applied Biosystems; Thermo Fisher Scientific, Inc.) at $37^{\circ} \mathrm{C}$ for $30 \mathrm{~min}$ and $84^{\circ} \mathrm{C}$ for $10 \mathrm{sec}$. $\mathrm{PPCR}$ was executed using the SYBR-Green Master Mix (Applied Biosystems; Thermo Fisher Scientific, Inc.) cycling profile: $95^{\circ} \mathrm{C}$ for $20 \mathrm{sec}, 40$ cycles of amplification $\left(95^{\circ} \mathrm{C}\right.$ for $30 \mathrm{sec}, 60^{\circ} \mathrm{C}$ for $30 \mathrm{sec}$ and $72^{\circ} \mathrm{C}$ for $30 \mathrm{sec}$ ) and melt curve analysis. The primer sequences for RT-qPCR: miR-30e 5'-GGGCAGTCT TTGCTACTGTAAAC-3' and 5'-GCCGCTGTAAACATC CGACT-3'; U6 5'-GCTTCGGCAGCACATATACTAAAA
T-3' and 5'-CGCTTCACGAATTTGCGTGTCAT-3'. The relative expression levels of microRNA-30e were calculated using the $2^{-\Delta \Delta C q}$ method (15).

Total RNA was hybridized using SurePrint G3 Mouse Whole Genome Microarray (G4471A-021828; Stratagene; Agilent Technologies, Inc., Santa Clara, CA, USA). Images were quantified using Agilent Feature Extraction Software (version A.10.7.3.1; Agilent Technologies, Inc.).

Cell culture, transfection and grouping. Human umbilical vein endothelial cells (HUVECs) were cultured in Dulbecco's modified Eagle's medium (DMEM; Thermo Fisher Scientific, Inc.) supplemented with $10 \%$ fetal bovine serum (Thermo Fisher Scientific, Inc.) at $37^{\circ} \mathrm{C}$ in $5 \% \mathrm{CO}_{2}$. In total, $100 \mathrm{ng}$ small interfering (si)-Nox4 (cat. no. sc-41586; Santa Cruz Biotechnology, Inc., Dallas, TX, USA), 100 ng microRNA-30e (5'-UGUAAACAUCCUUGACUGGAA G-3'), 100 ng anti-microRNA-30e ( 5'-UCCAGUCAAGGA UGUUUACAUU-3'), $100 \mathrm{ng}$ Snail plasmid (5'-CACTAT GCCGCGTCTTTCC-3'), 100 ng TGF- $\beta$ plasmid (5'-ACC CATGCCTCCCTCTCGGC-3') and $100 \mathrm{ng}$ negative mimics (5'-CCCCCCCCCCCCCC-3') were transfected into HUVECs $\left(1 \times 10^{5}\right.$ cells $\left./ \mathrm{ml}\right)$ using Lipofectamine ${ }^{\circledR} 2000$ (Thermo Fisher Scientific, Inc.). After transfection at $37^{\circ} \mathrm{C}$ for $6 \mathrm{~h}$, the medium was subsequently replaced with DMEM containing $10 \%$ fetal bovine serum for $42 \mathrm{~h}$ and treated with $500 \mu \mathrm{mol} / 1$ of $\mathrm{H}_{2} \mathrm{O}_{2}$ for $16 \mathrm{~h}$.

Luciferase reporter assays. The 3' untranslated region (UTR) of Snail was cloned into pMIR-REPORT luciferase reporter plasmids (Promega Corporation, Madison, WI, USA). Snai1 Plasmids and anti-microRNA-30e mimics were co-transfected into HUVECs using Lipofectamine ${ }^{\circledR} 2000$ (Thermo Fisher Scientific, Inc.). Following transfection at $37^{\circ} \mathrm{C}$ for $48 \mathrm{~h}$, cells were lysed using a dual luciferase reporter assay kit (Promega Corporation) according to the manufacturer's protocol. The absolute values of firefly luminescence were normalized to Renilla luciferase activity.

Western blotting. Total proteins from myocardial tissue were extracted using radioimmunoprecipitation buffer lysis buffer (Beyotime Institute of Biotechnology, Haimen, China) and protein contents were measured using bicinchoninic acid assay. A total of $50 \mu \mathrm{g}$ protein lysate of each sample was stacking on 4\% SDS-PAGE and transferred onto polyvinylidene difluoride membrane. The membrane was blocked with $5 \%$ skim milk powder at room temperature for $2 \mathrm{~h}$ and incubated with Snail (cat. no. sc-271977; 1:1,000), TGF- $\beta$ (cat. no. sc-52892; 1:1,000; both Santa Cruz Biotechnology, Inc.), Samd2 (cat. no. ab122028; 1:1,000), Nox4 (cat. no. ab133303; 1:1,000; both Abcam, Cambridge, USA) and GAPDH (cat. no. sc-293335; 1:5,000; Santa Cruz Biotechnology, Inc.) at $4^{\circ} \mathrm{C}$ overnight. On the next day, membrane was washed with Tris buffered saline $0.1 \%$ Tween 20 for three times and incubated with horseradish peroxidase labeled secondary antibody (cat. no. sc-2004; 1:5,000; Abcam) at room temperature for $1 \mathrm{~h}$. The absorbance of each band was analyzed using Image Lab 3.0 (Bio-Rad Laboratories, Inc., Hercules, CA, USA) following enhanced chemiluminescence (cat. no. P0018AS; Beyotime Institute of Biotechnology) staining. 
A

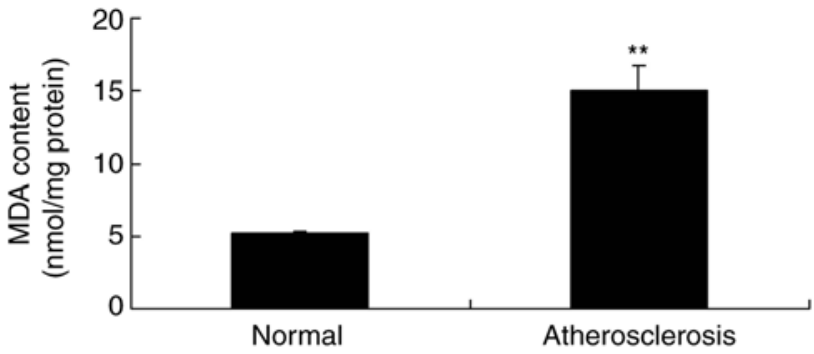

C

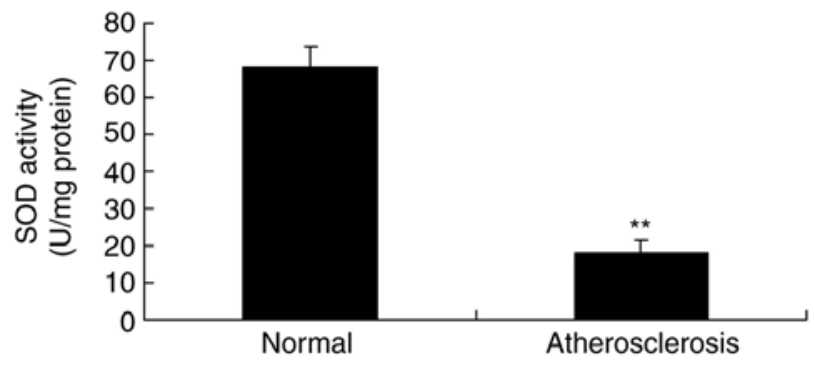

$E$

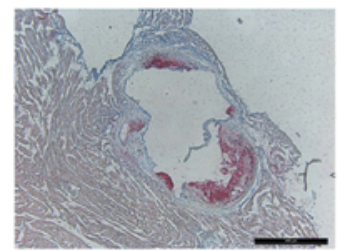

Normal

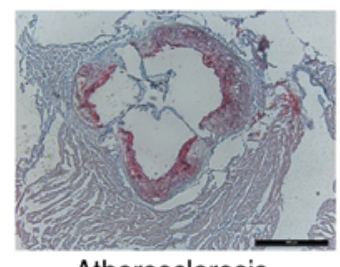

Atherosclerosis

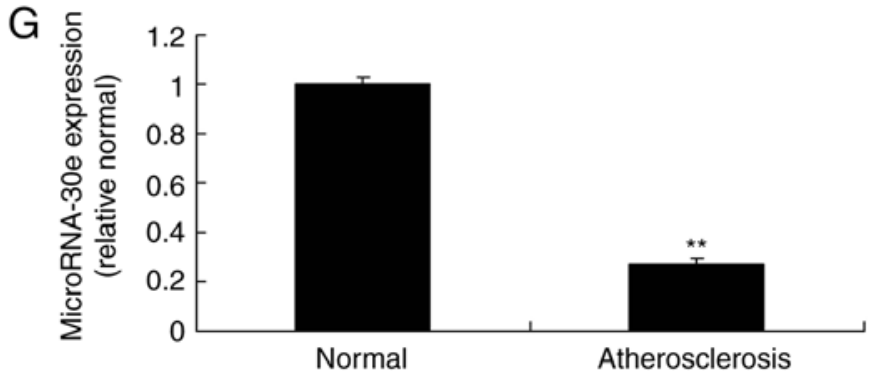

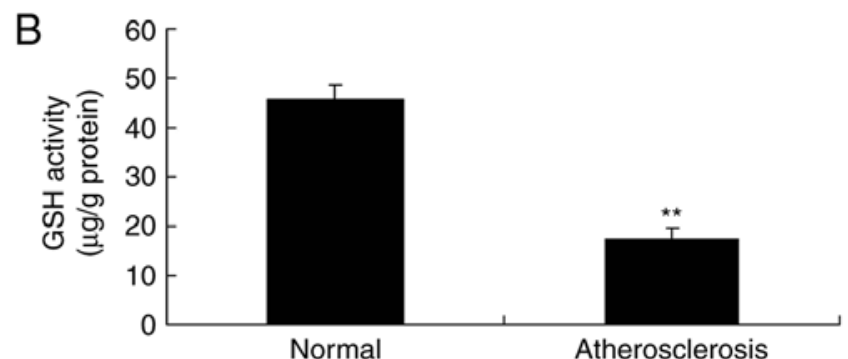

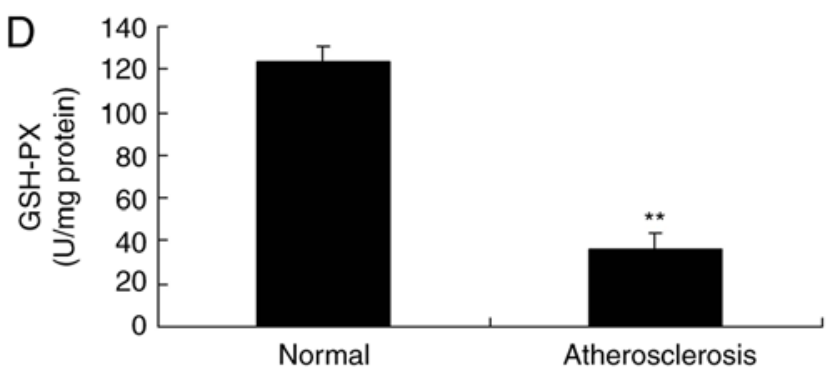

$\mathrm{F}$

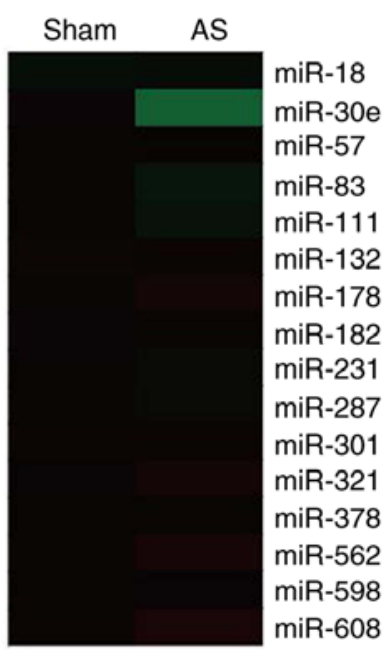

Figure 1. Expression of miRNA-30e in an atherosclerosis model. (A) MDA, (B) SOD, (C) GSH and (D) GSH-PX, (E) Oil Red O staining, (F) heat map and $(\mathrm{G})$ quantitative polymerase chain reaction for miRNA-622 expression. Scale bar, $400 \mu \mathrm{m}$. ${ }^{* *} \mathrm{P}<0.01$ vs. the control normal group. Normal, control normal group; Atherosclerosis, Atherosclerosis model group; miR/miRNA, microRNA; GSH-PX, glutathione-peroxidase; MDA, malondiadehyde; SOD, superoxide.

Statistical analysis. All the data are expressed as mean \pm standard error of the mean $(n=3)$. Statistical analysis was performed using SPSS 17.0 (SPSS, Inc., Chicago, IL, USA). Student's t-test or one-way analysis of variance and Tukey's post hoc test was used to analyze comparisons. $\mathrm{P}<0.05$ was considered to indicate a statistically significant difference.

\section{Results}

Expression of miRNA-30e in an AS model. The effects and mechanisms of miRNA-30e in were first evaluated in an AS model. MDA levels were significantly increased, while the levels of SOD, GSH and GSH-PX were significantly decreased in mice with AS, compared with the normal control group $(\mathrm{P}<0.01$; Fig. 1A-D). As presented in Fig. 1E, the number of thrombi in mice of AS was increased compared with the normal control group. The expression of miRNA-30e in the AS model group was significantly reduced, compared with the normal mice group $(\mathrm{P}<0.01$; Fig. $1 \mathrm{~F}-\mathrm{G})$. In conclusion, these results demonstrated that miRNA-30e may be involved in AS.

Downregulation of miRNA-30e increases oxidative stress and $R O S$. To investigate the function of miRNA-30e downregulation in AS, anti-miRNA-30e was used to reduce the expression of miRNA-30e. As presented in Fig. 2A, anti-miRNA-30e significantly inhibited the expression of miRNA-30e in an in vitro model, compared with the negative group $(\mathrm{P}<0.01)$. Downregulation of miRNA-30e significantly increased MDA levels, while the levels of SOD, GSH and GSH-PX were significantly decreased, and significantly promoted ROS levels in an in vitro model, compared with the negative group ( $\mathrm{P}<0.01$; Fig. 2B-G).

Over-expression of miRNA-30e reduces oxidative stress and $R O S$. Then an miRNA-30e mimics was used to increase the 

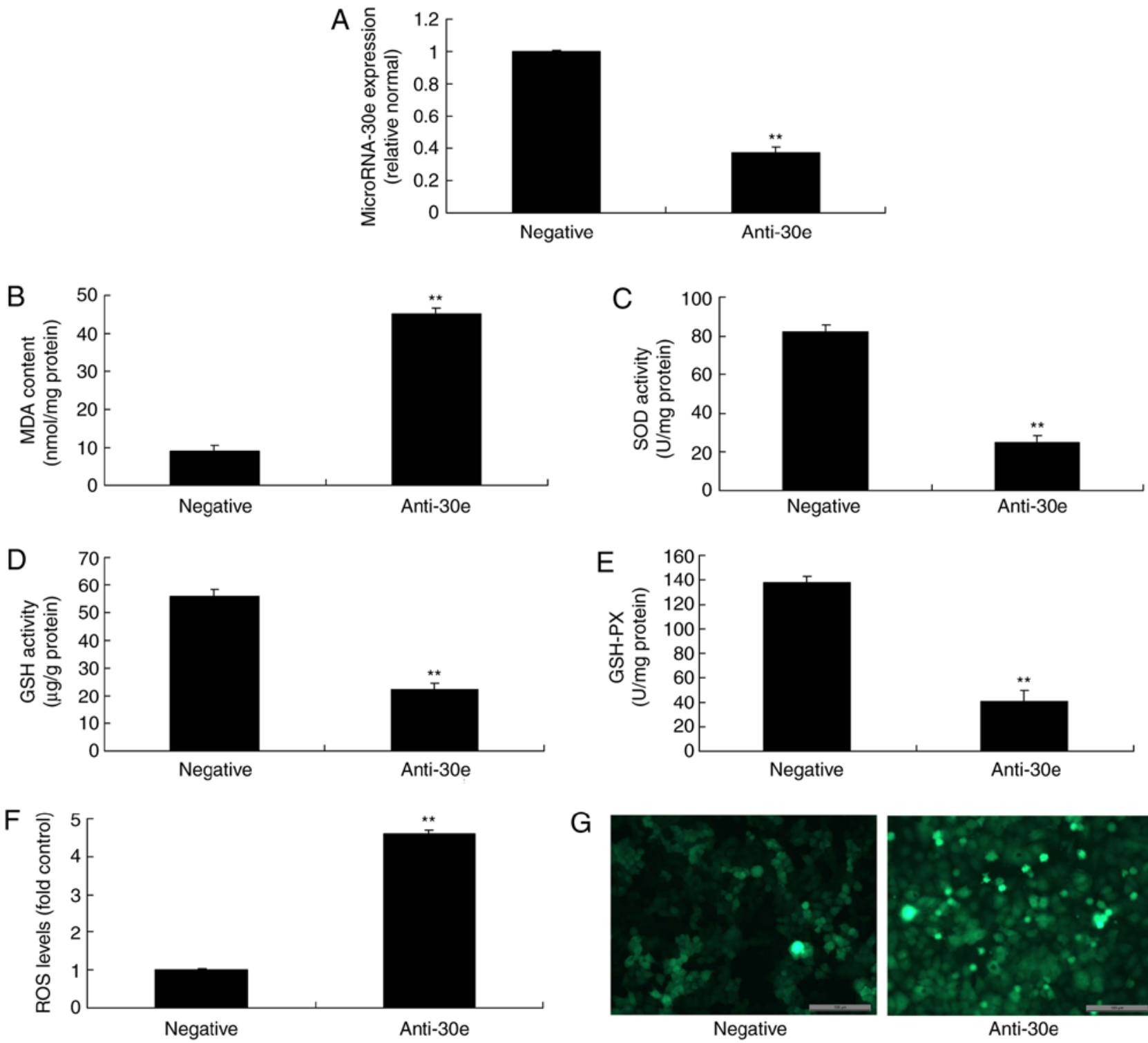

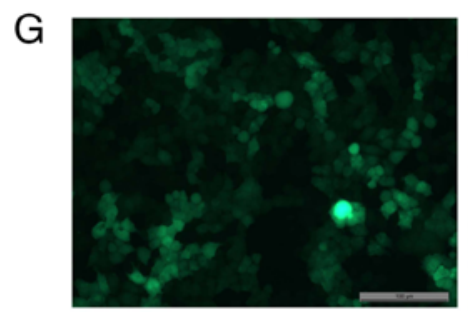

Negative

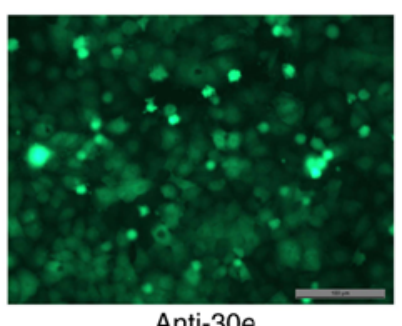

Anti-30e

Figure 2. Downregulation of miRNA-30e increases oxidative stress and ROS. (A) miRNA-30e expression, (B) MDA, (C) SOD, (D) GSH and (E) GSH-PX, (F) ROS levels and $(\mathrm{G})$ green fluorescent protein staining of cells following anti-miRNA30e transfection. Scale bar, $100 \mu \mathrm{m}$. ${ }^{* *} \mathrm{P}<0.01 \mathrm{vs}$. the negative normal group. miR/miRNA, microRNA; GSH-PX, glutathione-peroxidase; MDA, malondiadehyde; SOD, superoxide; ROS, reactive oxygen species; Negative, negative control group; anti-30e, downregulation of microRNA-30e group.

expression of miRNA-30e in an in vitro model, compared with the negative group (Fig. 3A). Over-expression of miRNA-30e reduced MDA levels, increased the levels of SOD, GSH and GSH-PX, and decreased ROS levels in an in vitro model, compared with the negative group (Fig. 3B-G). Therefore, it was concluded that miRNA-30e regulated ROS and oxidative stress in AS.

miRNA-30e regulates TGF- $\beta$-mediated Nox4-dependent oxidative stress by Snail. Furthermore, the mechanism of miRNA-30e on ROS and oxidative stress was analyzed in AS. The results of the Gene chip demonstrated that downregulation of miRNA-30e induced the expression of Snail in vitro, compared with the negative group (Fig. 4A). Snai1 was a putative target of miRNA-30e, confirmed by Luciferase reporter activity which was significantly increased in an in vitro model, compared with the negative group $(\mathrm{P}<0.01$; Fig. 4B and $\mathrm{C})$.
Downregulation of miRNA-30e significantly induced the expression of the Snail protein in an in vitro model, compared with the negative group $(\mathrm{P}<0.01$; Fig. 4D).

As presented in Fig. 4E-I, downregulation of miRNA-30e induced the protein expression of Snai1, TGF- $\beta$ and Smad2 and suppressed Nox4 protein expression in an in vitro model, compared with the negative group. In contrast, over-expression of miRNA-30e significantly suppressed the protein expression of Snai1, TGF- $\beta$ and Smad2 $(\mathrm{P}<0.01)$ and significantly induced that of Nox4 in an in vitro model, compared with the negative group $(\mathrm{P}<0.01$; Fig. 5$)$. These results demonstrated that miRNA-30e regulated Snail/TGF- $\beta /$ Nox 4 protein expression to affect ROS/oxidative stress in $\mathrm{AS}$.

The activation of Snail attenuates the effects of miRNA-30e on oxidative stress in vitro. Therefore, to further evaluate 

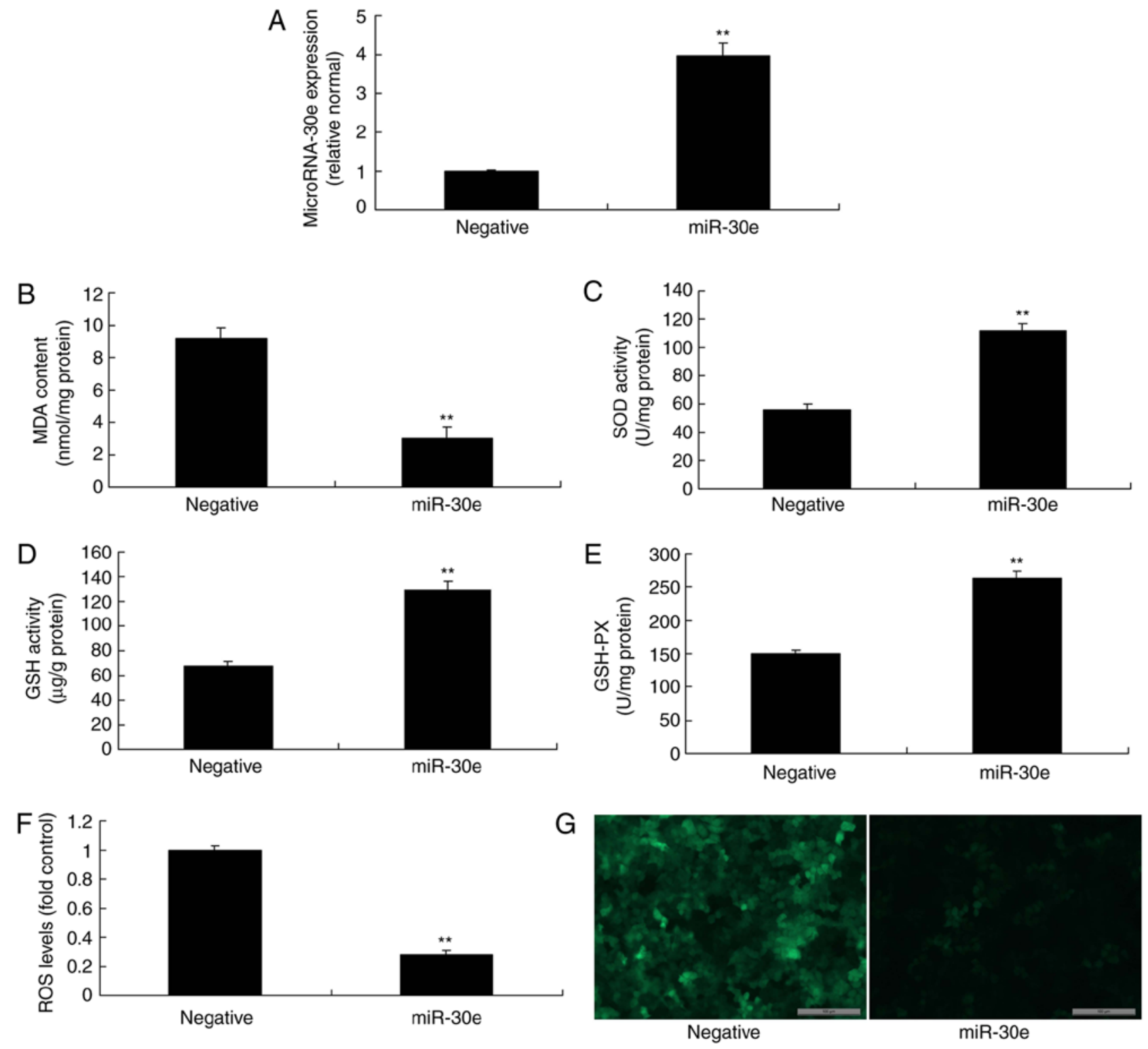

Figure 3. Overexpression of miRNA-30e reduces oxidative stress and ROS. (A) miRNA-30e expression, (B) MDA, (C) SOD, (D) GSH and (E) GSH-PX, (F) ROS levelsand (G) green fluorescent protein staining of cells following anti-miRNA30e transfection. Scale bar, $100 \mu \mathrm{m}$. ** P $<0.01 \mathrm{vs.} \mathrm{the} \mathrm{negative} \mathrm{normal}$ group. miR/miRNA, microRNA; GSH-PX, glutathione-peroxidase; MDA, malondiadehyde; SOD, superoxide; ROS, reactive oxygen species; Negative, negative control group; miR-30e, over-expression of microRNA-30e group.

the role of Snail in the effects of miRNA-30e on oxidative stress in an in vitro model, a Snail plasmid was used to significantly increase the protein expression of Snai1, TGF- $\beta$ and Smad2 $(\mathrm{P}<0.01)$ and significantly suppressed that of Nox4 in an in vitro model by miRNA-30e, compared with the miRNA-30e group ( $\mathrm{P}<0.01$; Fig. 6A-E). The activation of Snail significantly attenuated the effects of miRNA-30e on the inhibition of MDA and ROS levels, and activation of SOD, GSH and GSH-PX levels in an in vitro model, compared with the miRNA-30e group $(\mathrm{P}<0.01$; Fig. 6F-K). Snail is a target spot for the effects of miRNA-30e on oxidative stress in AS.

The activation of TGF- $\beta$ attenuates the effects of miRNA-30e on oxidative stress in an in vitro model. Next, the function of TGF- $\beta$ in the effects of miRNA-30e on oxidative stress in an in vitro model was investigated further. To this end, a TGF- $\beta$ plasmid significantly induced the protein expression of TGF- $\beta$, Smad 2 and significantly suppressed Nox 4 in an in vitro model following miRNA-30e, compared with the miRNA-30e group $(P<0.01$; Fig. 7A-D). Additionally, the activation of TGF- $\beta$ attenuated the effects of miRNA-30e on the inhibition of MDA and ROS levels, and activation of SOD, GSH and GSH-PX levels in an in vitro model, compared with the microRNA-30e group (Fig. 7E-K). These results support that TGF- $\beta$ is an important player for the effects of miRNA-30e on oxidative stress in AS.

The inhibition of Nox4 attenuates the effects of miRNA-30e on oxidative stress in an in vitro model. Finally, si-Nox4 could significantly suppress the protein expression of Nox4 in an in vitro model following miRNA-30e transfection, compared with the miRNA-30e group $(\mathrm{P}<0.01$; Fig. 8A). Then, the inhibition of Nox4 significantly attenuated the 

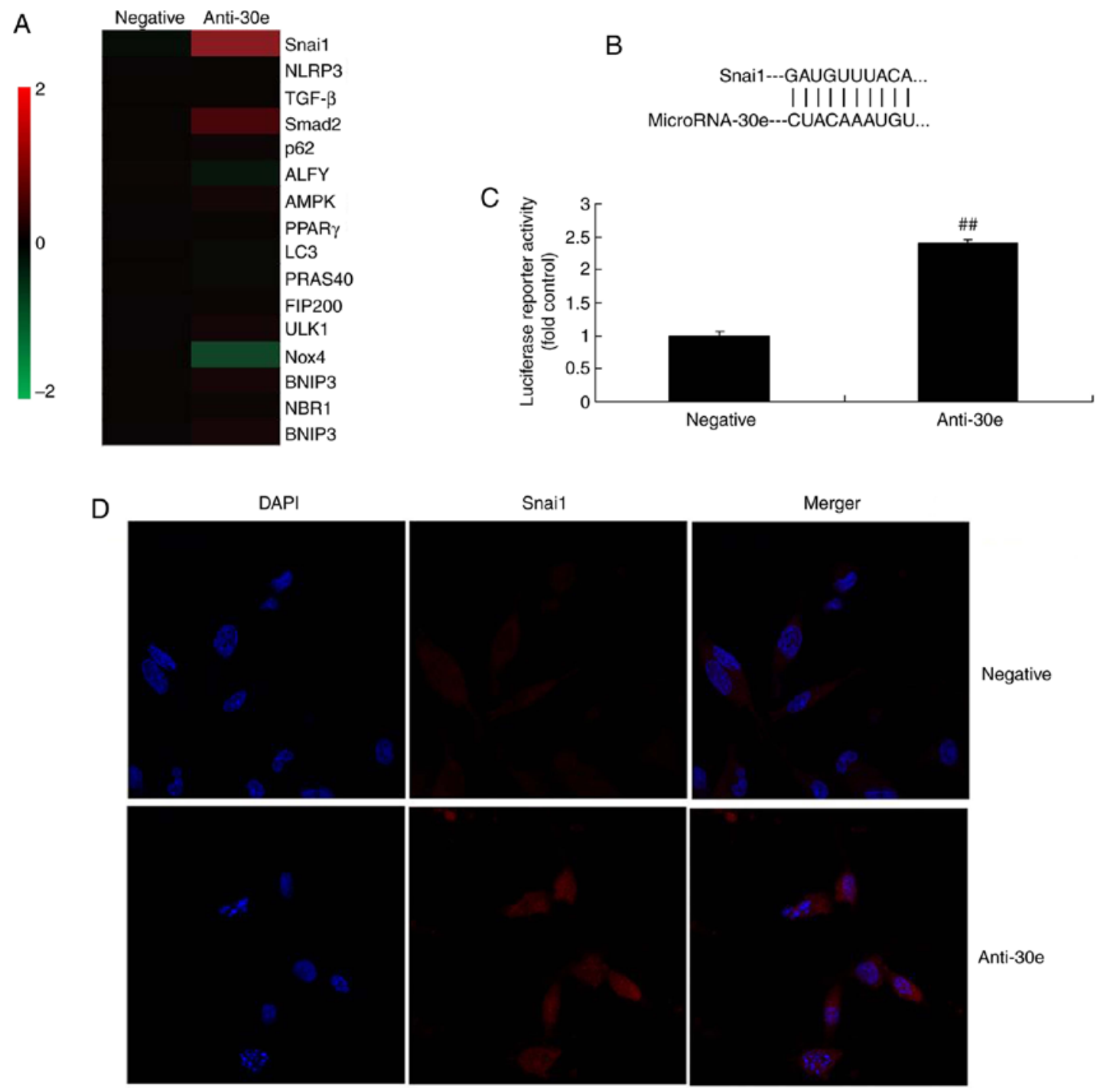

\section{E}
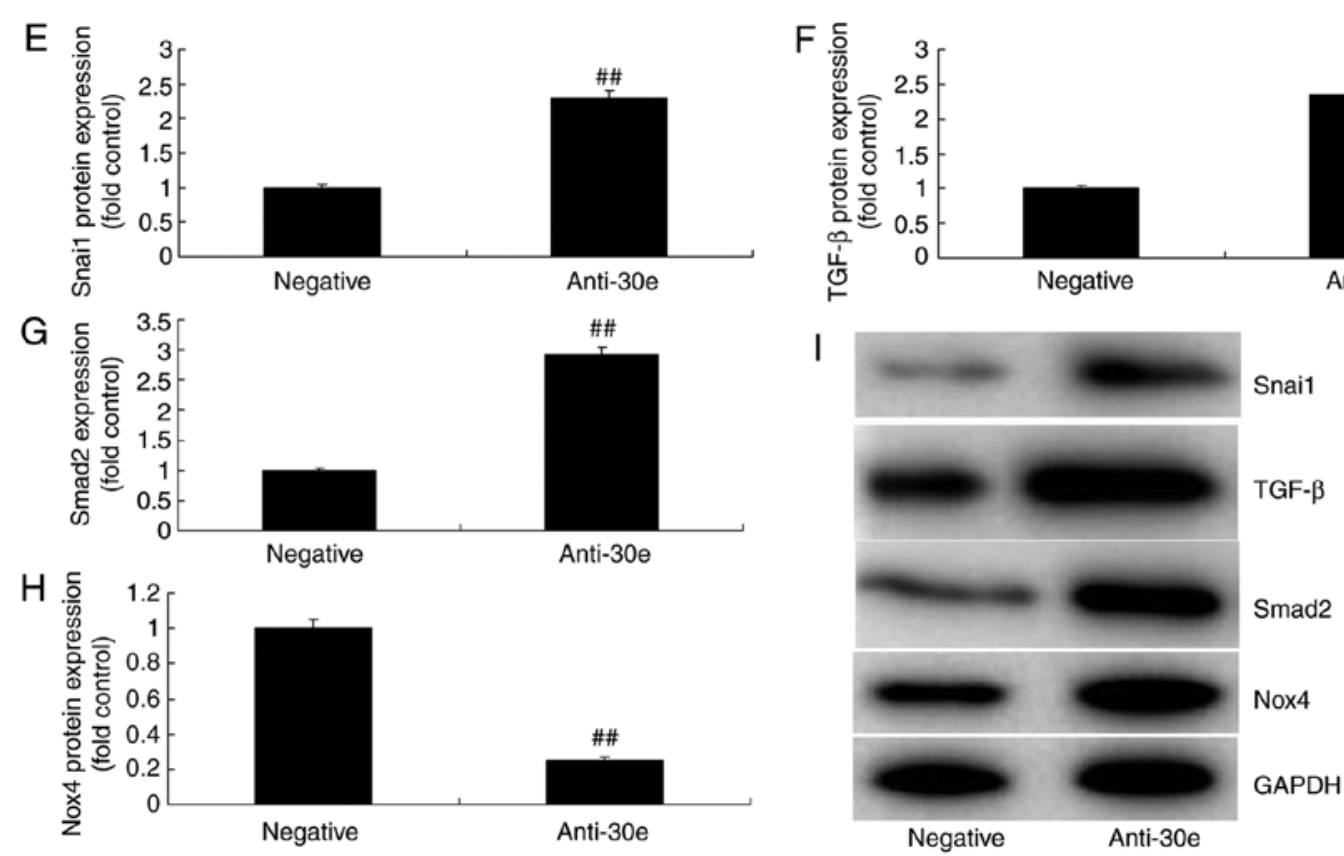

Figure 4. miRNA-30e regulates TGF- $\beta$-mediated NADPH oxidase 4-dependent oxidative stress by Snail. (A) Heat map for signaling pathway, (B) Snai1 was a putative target of miRNA-30e and (C) luciferase reporter activity, (D) Snail protein expression. (E) Snail, (F) TGF- $\beta$, (G) Smad2 and (H) Nox4 protein expression by statistical analysis, and (I) western blotting analysis. ${ }^{\# \#} \mathrm{P}<0.01$ vs. the negative normal group. miR/miRNA, microRNA; TGF, transforming growth factor; Nox4, NAPDH oxidase 4; Smad, mothers against decapentaplegic homolog; negative, negative control group; anti-30e, downregulation of microRNA-30e group. 

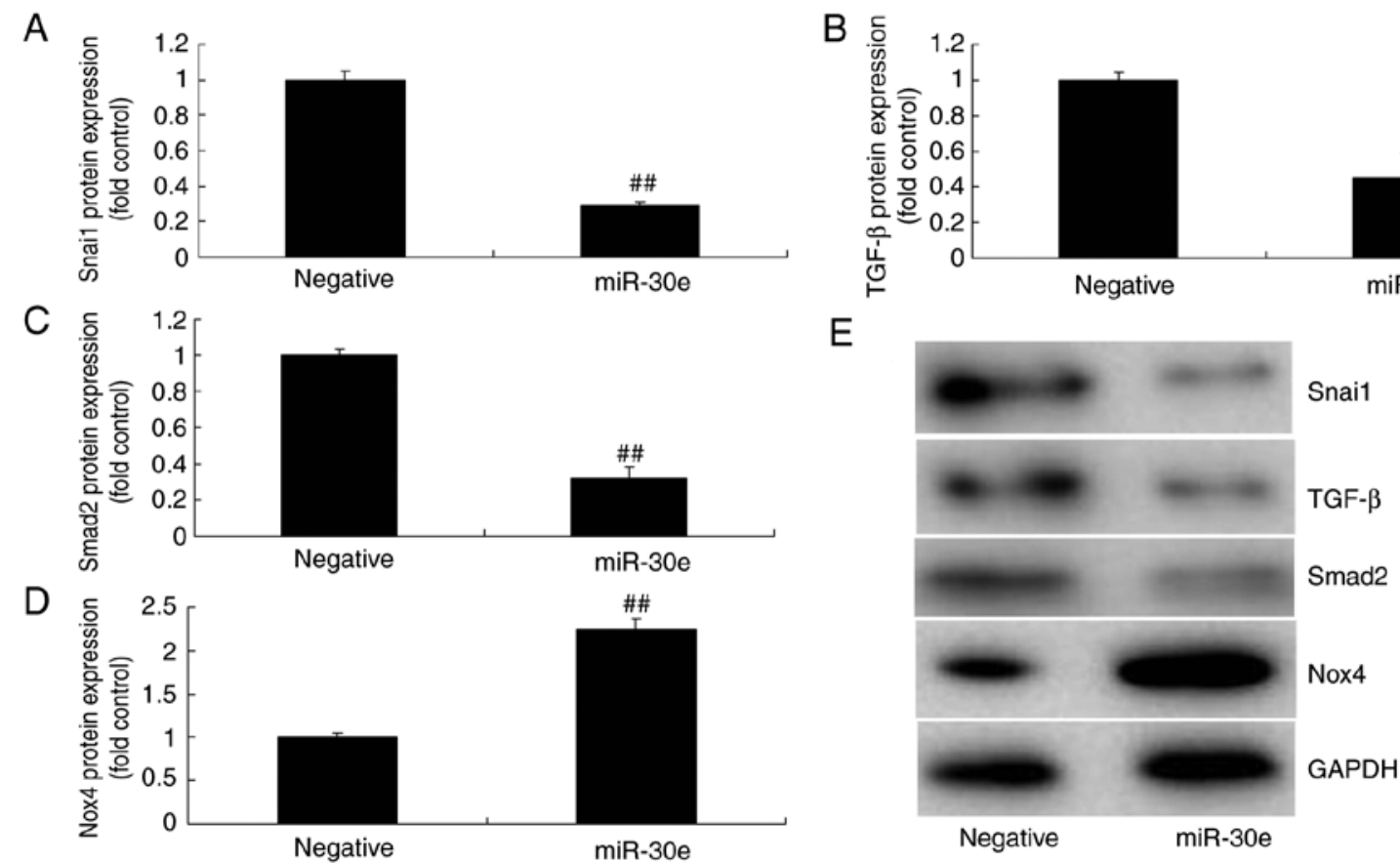

Figure 5. miRNA-30e regulates Snai1/TGF- $\beta /$ Nox4 protein expression. (A) Snai1, (B) TGF- $\beta$, (C) Smad2 and (D) Nox4 protein expression by statistical analysis, and (E) western blotting analysis. ${ }^{\# \#} \mathrm{P}<0.01$ vs. the negative normal group. miR/miRNA, microRNA; TGF, transforming growth factor; Nox4, NAPDH oxidase 4; Smad, mothers against decapentaplegic homolog; Negative, negative control group; miR-30e, overexpression of microRNA-30e group.

effects of miRNA-30e on the inhibition of MDA and ROS levels, and activation of SOD, GSH and GSH-PX levels in an in vitro model, compared with the miRNA-30e group without Nox4 inhibition $(\mathrm{P}<0.01$; Fig. $8 \mathrm{~B}-\mathrm{G})$. In conclusion, the inhibition of Nox 4 attenuated the effects of miRNA-30e on oxidative stress in vitro.

\section{Discussion}

Angiogenesis is a vital influencing factor during AS plaque development. Local angiogenesis is correlated with plaque stability (16). The increasing angiogenesis in the plaque will accumulate lipid and various inflammatory cells within the plaque. Finally, it will result in matrix degradation, fibrous cap thinning, plaque rupture and therefore induce a severe cardiovascular event (17). miRNA research suggests that miRNA serves a key role in the fields of tumor disease and AS. Recently, multiple AS-associated miRNAs have been identified in the cardiovascular system, which may provide a novel clue for the early diagnosis and treatment selection for AS (18). The results of the present study demonstrated that the expression of miRNA-30e in the AS model group was reduced, compared with the normal mice group. Lai et al (14) demonstrated that miRNA-30e mediated cardioprotection of ACE2 in rats with doxorubicin-induced heart failure.

$\mathrm{OS}$ is present in the whole process of $\mathrm{AS}$, from fat lesion formation to plaque rupture. Furthermore, it mediates the functional alterations and injury of vascular endothelial cells, smooth muscle cells and mononuclear macrophages (19). Therefore, it is of great significance to illustrate the molecular mechanism of cellular OS in AS genesis and development. This is important to delay AS progression and prevent the incidence of acute cardiovascular and cerebrovascular events (19).
In this study, it was demonstrated that the downregulation of miRNA-30e increased MDA levels and reduced SOD, GSH and GSH-PX levels in vitro model. Jin et al (20) reported that miR-30e-UCP2 pathway regulates alcoholic hepatitis progress through suppression of oxidative stress.

Current research suggests that ROS may induce AS genesis and development through the following mechanisms (21). ROS can react with the multivalent unsaturated fatty acid in the biomembrane and therefore directly induce lipid peroxidation in the biomembrane. As a result, a large amount of lipid peroxides and aldehydes will be produced, therefore increasing the membrane permeability and causing tissue injury. ROS can promote the degeneration of intracellular proteins and enzymes, and cause protein function loss and enzyme inactivation. In addition, it can destroy nucleic acids and chromosomes, resulting in DNA strand breaking, chromosome mutation or rupture (21). Furthermore, ROS can affect the redox status and ion channel of cells, causing protein nitration or inactivation. Therefore, it will restrict mitochondrial respiration, promote endothelial cell degeneration, necrosis and apoptosis, and destroy the vascular endothelial integrity. In the present study, the downregulation of miRNA-30e promoted ROS levels in the in vitro model. These results demonstrated that the downregulation of miRNA-30e promoted oxidative stress and ROS levels in vitro model of AS.

TGF- $\beta$ is a multi-functional protein associated with the development and trauma repair of tissues. TGF- $\beta$ participates in immune function regulation and tumor genesis in the body. There are 3 subtypes in the human body, which share similar biological characteristics. Among them, TGF- $\beta 1$ is present in the greatest proportion and has the highest activity (21). It mainly exerts its regulatory role 
A

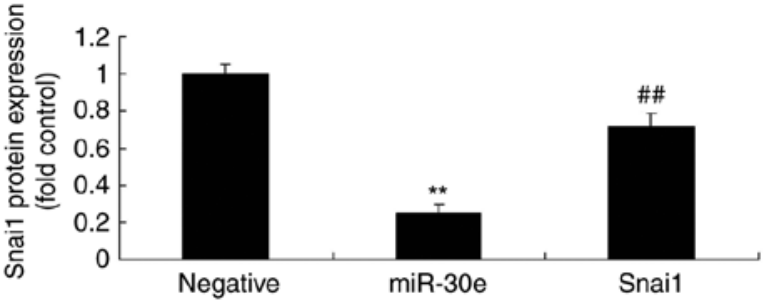

C

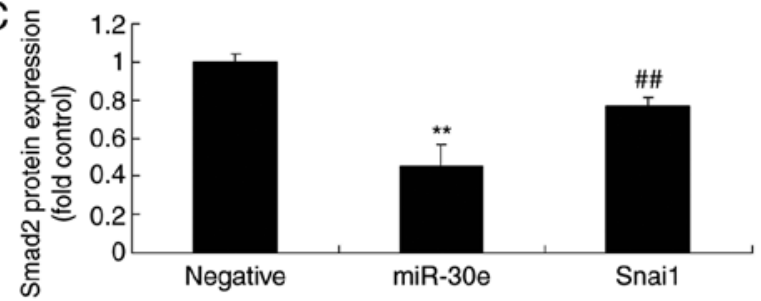

D

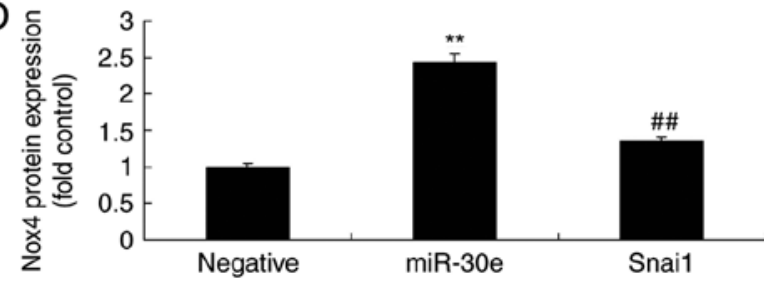

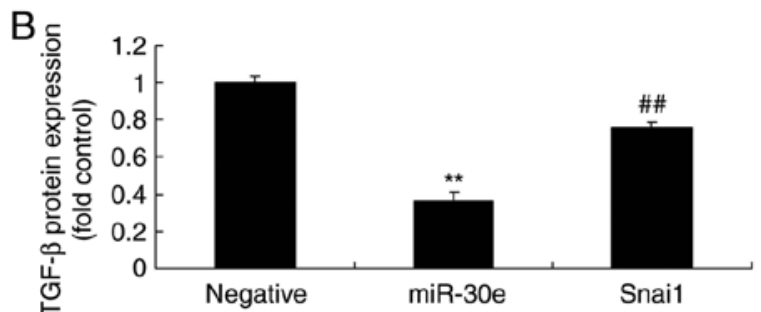

E

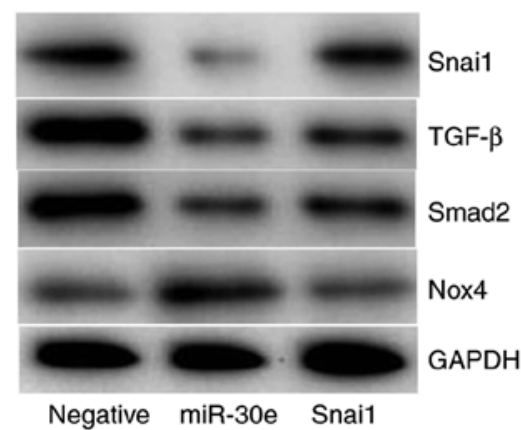

Negative miR-30e Snai1
$\mathrm{F}$

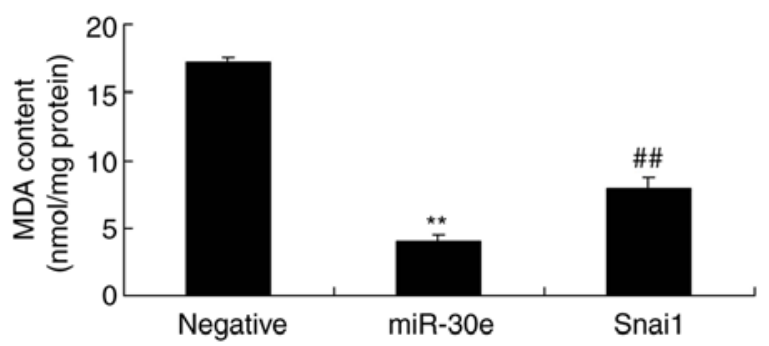

$\mathrm{H}$

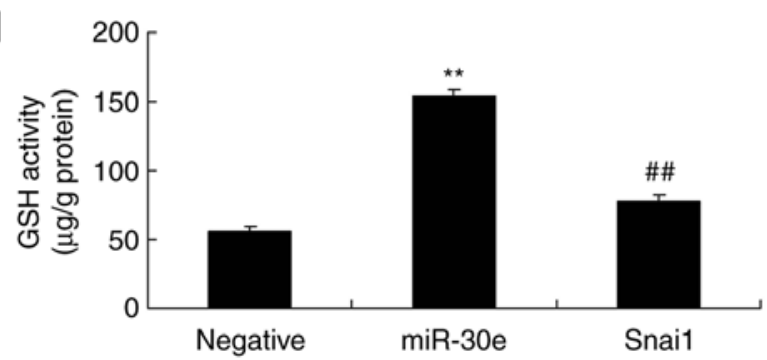

G

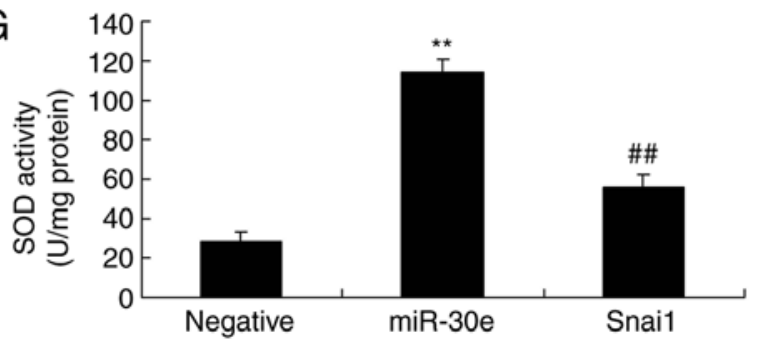

I

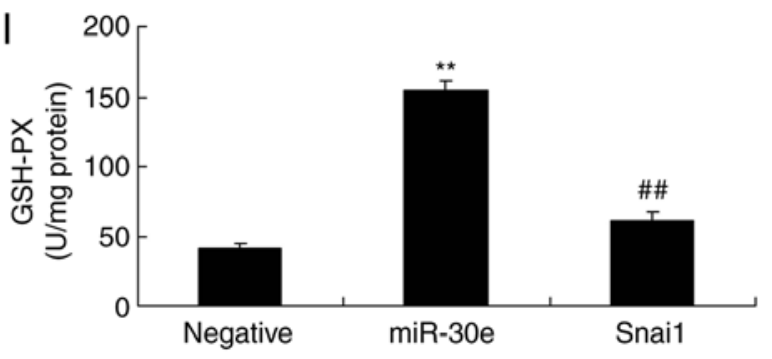

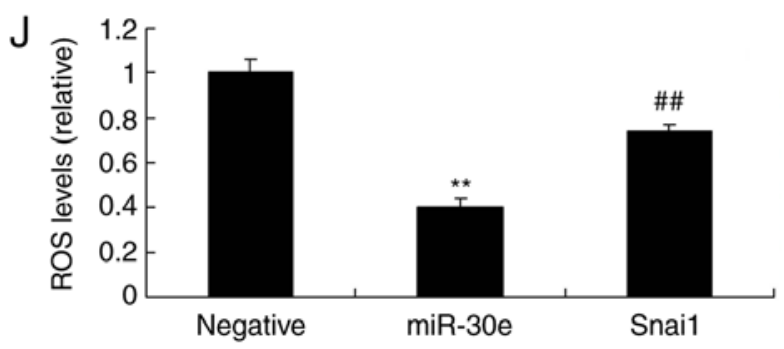

$\mathrm{K}$

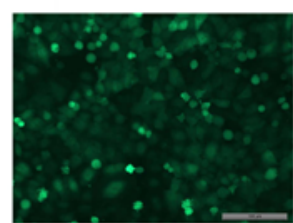

Negative

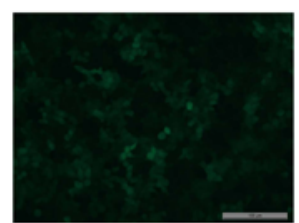

miR-30e

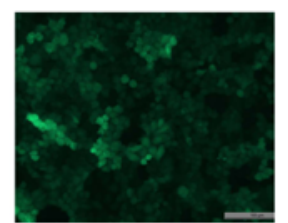

Snai1

Figure 6. Activation of Snai1 reduces the effects of miRNA-30e on the oxidative stress in vitro model. (A) Snai1, (B) TGF- $\beta$, (C) Smad2 and (D) Nox4 protein expression by statistical analysis, and (E) western blotting analysis, (F) MDA, (G) SOD, (H) GSH and (I) GSH-PX, (J) ROS levels and (K) green fluorescent protein transfection. Scale bar, $100 \mu \mathrm{m}$. ${ }^{* *} \mathrm{P}<0.01$ vs. the negative normal group, ${ }^{\# \#} \mathrm{P}<0.01$ vs. over-expression of miRNA-30e group. miR/miRNA, microRNA; TGF, transforming growth factor; Nox4, NAPDH oxidase 4; Smad, mothers against decapentaplegic homolog; Negative, negative control group; miR-30e, over-expression of miRNA-30e group; Snai1, over-expression of miRNA-30e and Snai1 plasmid group.

through autocrine and paracrine routes involving the cells of the vascular wall including smooth muscle cells and vascular endothelial cells. These results suggest that downregulation of miRNA-30e induced Snai1, TGF- $\beta$ and Smad2 protein expression and suppressed Nox4 protein expression in an in vitro model. Zhang et al (22) demonstrated that miR-30e attenuates isoproterenol-induced cardiac fibrosis via Snai1/TGF- $\beta$. 

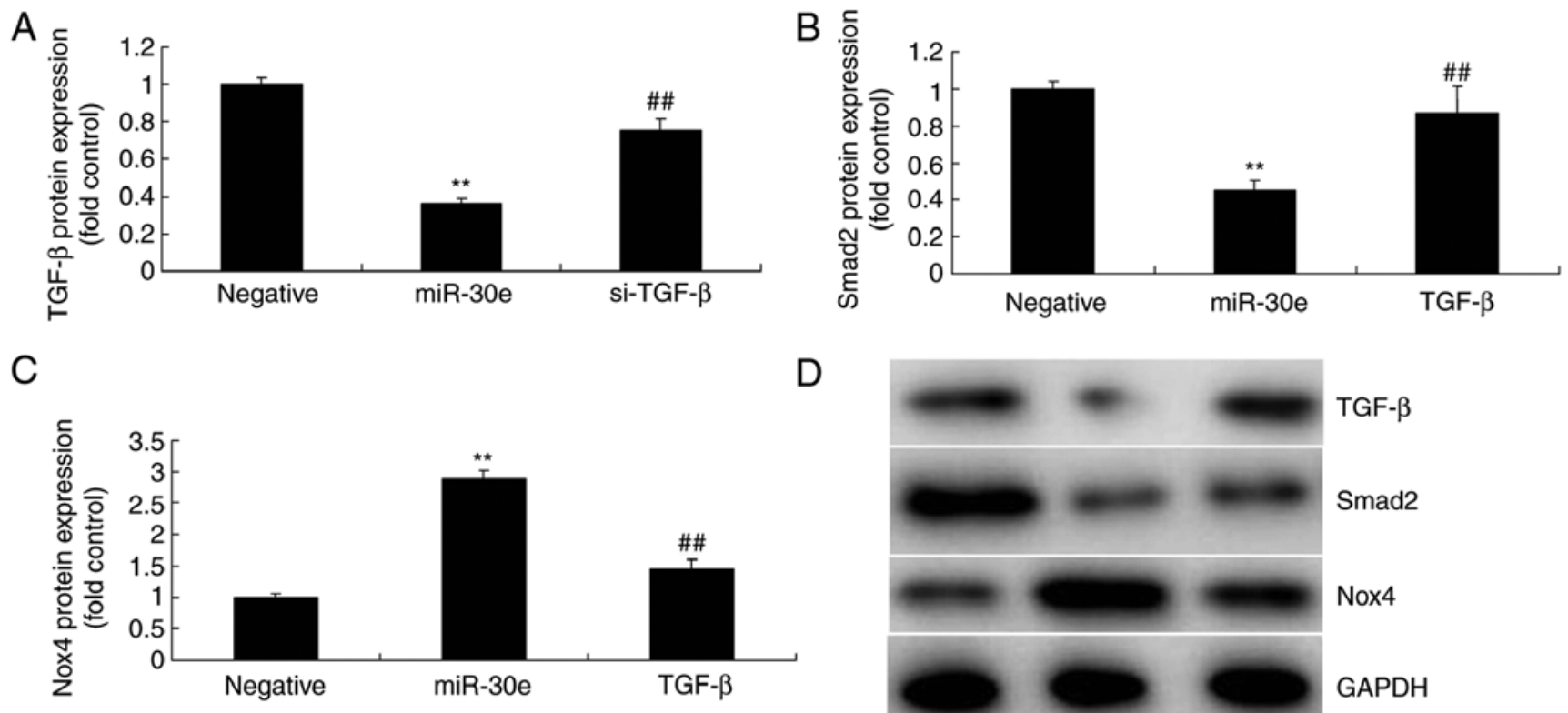

D
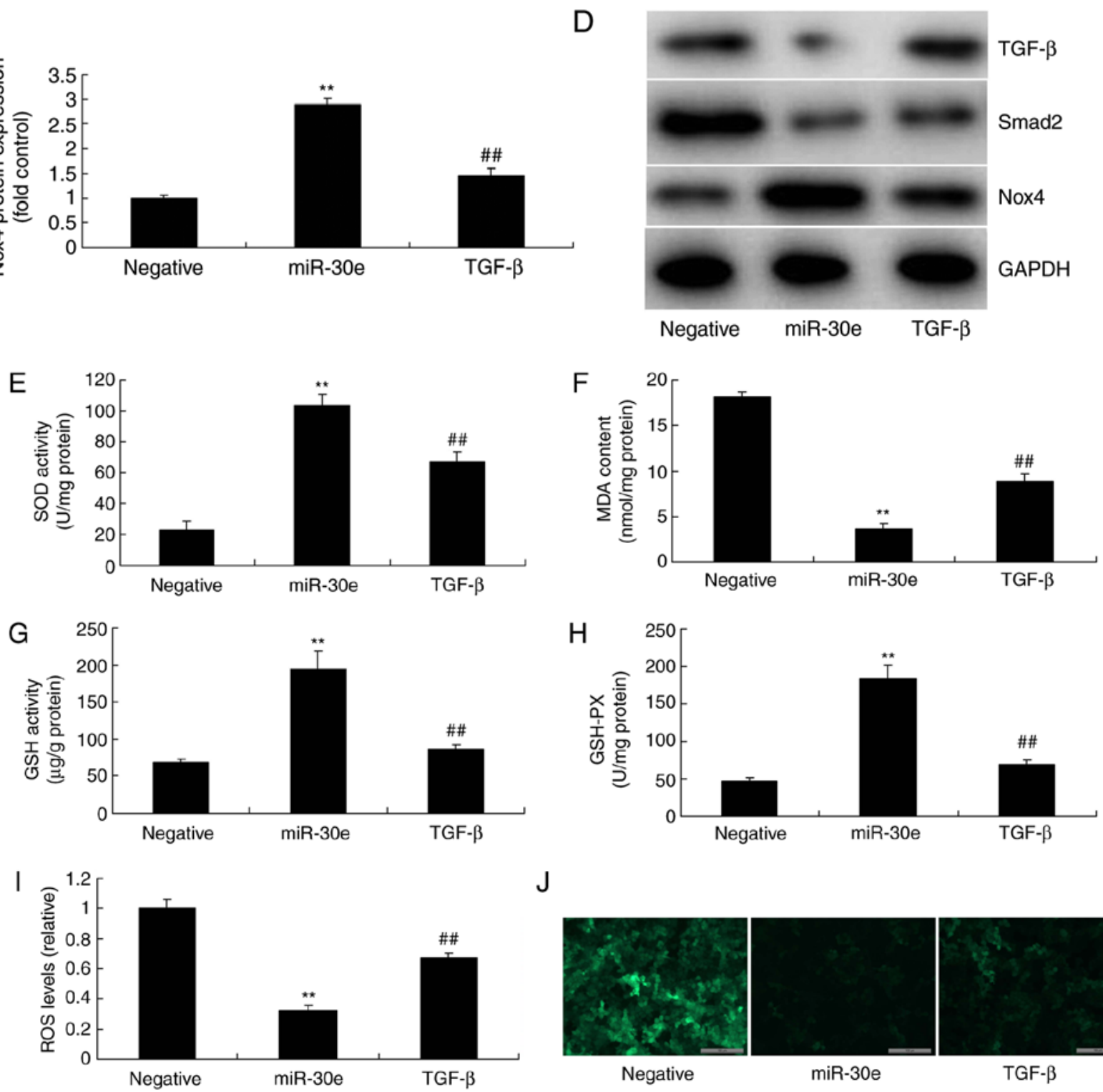

$J$

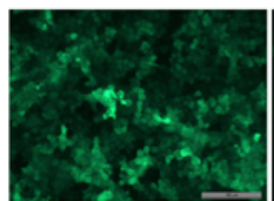

Negative

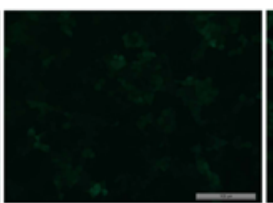

miR-30e

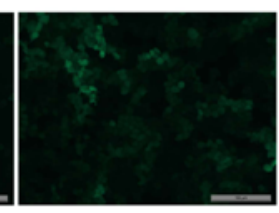

TGF- $\beta$

Figure 7. Activation of TGF- $\beta$ reduces the effects of miRNA-30e on the oxidative stress in vitro model. (A) TGF- $\beta$, (B) Smad 2 and (C) Nox 4 protein expression by statistical analysis and (D) western blotting analysis, (E) MDA, (F) SOD, (G) GSH and (H) GSH-PX (I) ROS levels and (J) green fluorescent protein. ${ }^{* *} \mathrm{P}<0.01$ vs. the negative normal group, ${ }^{\# \#} \mathrm{P}<0.01$ vs. the over-expression of microRNA-30e group. miR/miRNA, microRNA; TGF, transforming growth factor; Nox4, NAPDH oxidase 4; Smad, mothers against decapentaplegic homolog; Negative, negative control group; miR-30e, over-expression of microRNA-30e group; TGF- $\beta$, over-expression of microRNA-30e and TGF- $\beta$ plasmid group.

OS-induced ROS accumulation serves a vital role in AS genesis and development. Nox is a major enzyme in ROS production in blood vessels, which takes part in AS genesis and development (11). Studies report that exogenous angiotensin (Ang)II can increase the Nox-origin ROS production in smooth muscle cells, which will therefore damage their structure and induce vascular remodeling (12). Exogenous AngII will upregulate the peroxidasin homolog protein and mRNA expression in smooth muscle cells, accompanying the increase in intracellular $\mathrm{H}_{2} \mathrm{O}_{2}$ (23). Therefore, miRNA-30e regulates Snai1/TGF- $\beta /$ Nox 4 to reduce oxidative stress in an in vitro model of AS.

In conclusion, it was demonstrated that the expression of microRNA-30e was reduced in an AS model and 
A

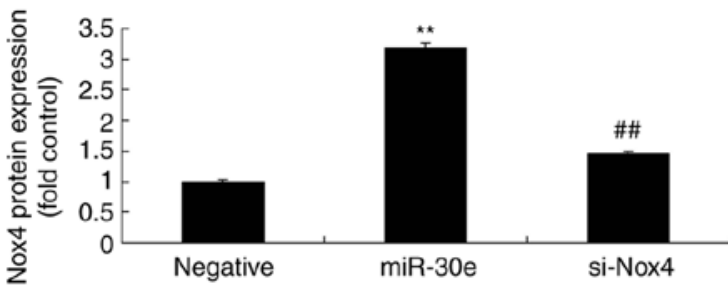

C

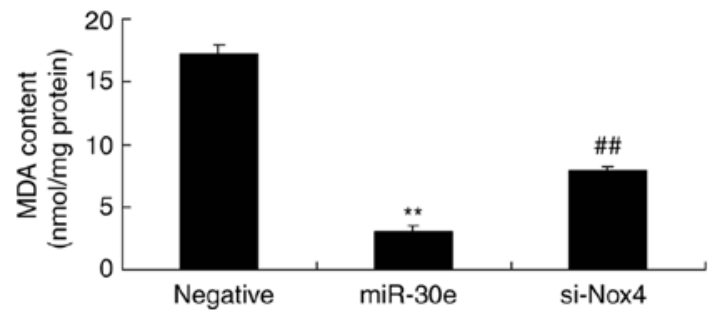

$\mathrm{E}$

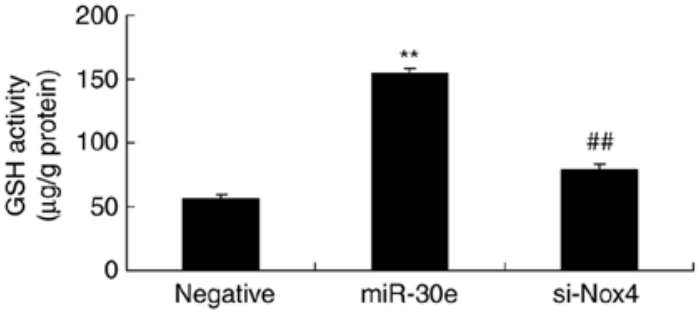

B
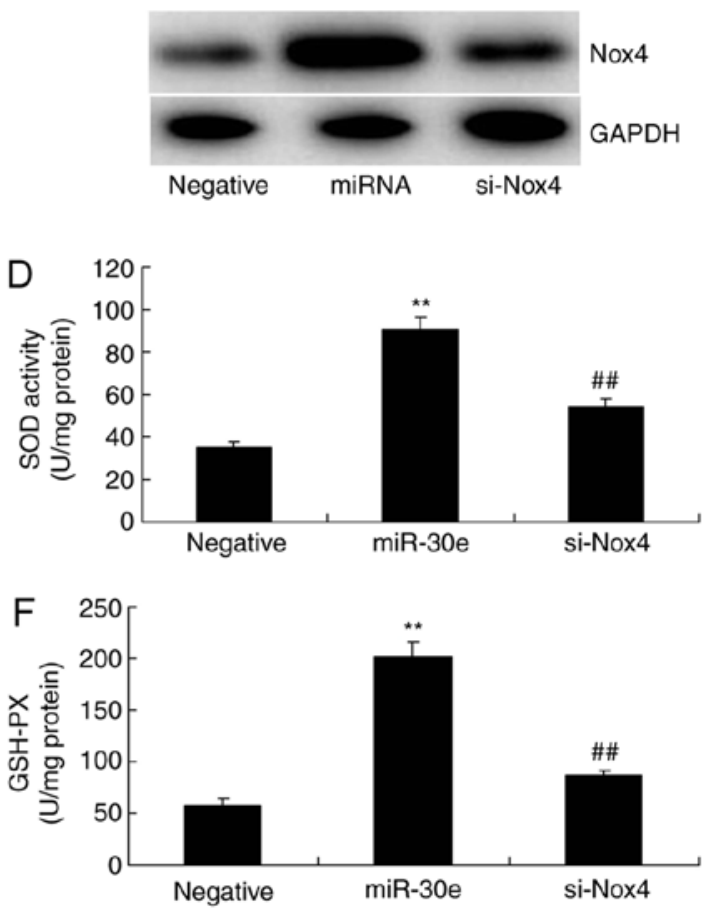

G

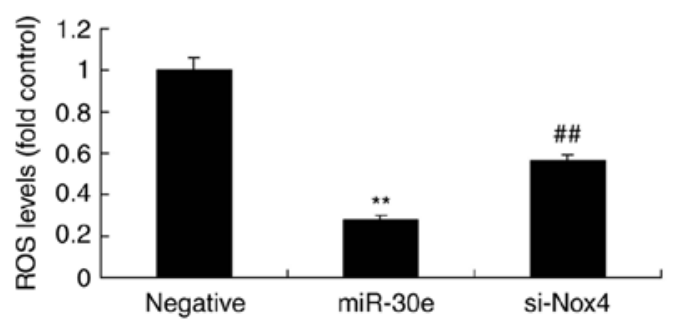

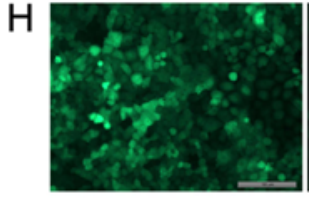

Negative

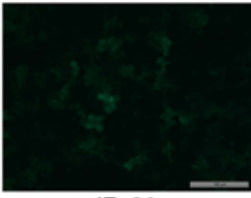

miR-30e

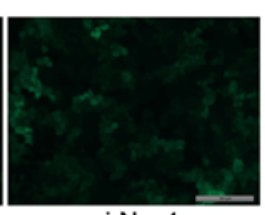

si-Nox4

Figure 8. Inhibition of Nox4 reduces the effects of miRNA-30e on the oxidative stress in vitro model. Nox4 protein expression by (A) statistical analysis and (B) western blotting analysis, (C) MDA, (D) SOD, (E) GSH and (F) GSH-PX, (G and H) ROS levels. Negative, negative control group; miR-30e, over-expression of microRNA-30e group; si-Nox4, over-expression of microRNA-30e and si-Nox4 group. ${ }^{* * *} \mathrm{P}<0.01$ vs. the negative normal group, ${ }^{\# \#} \mathrm{P}<0.01$ vs. the over-expression of miRNA-30e group. miR/miRNA, microRNA; TGF, transforming growth factor; Nox4, NAPDH oxidase 4; Smad, mothers against decapentaplegic homolog.

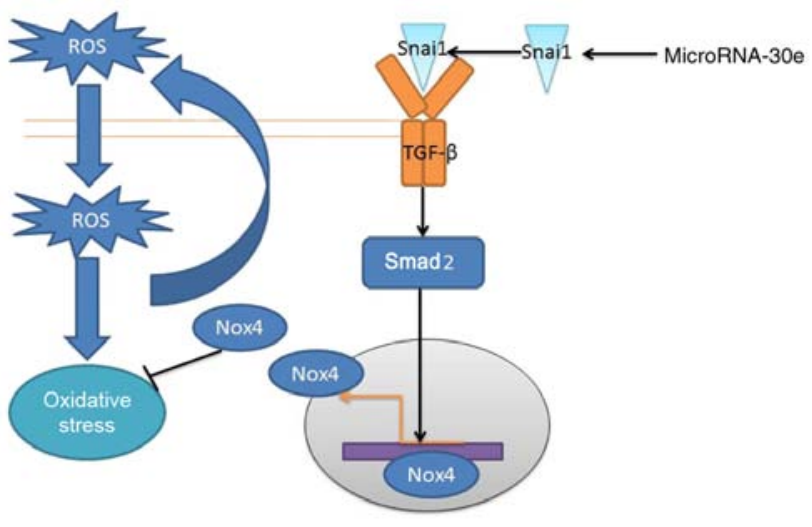

Figure 9. MicroRNA-30e regulates atherosclerosis by TGF- $\beta$-mediated Nox 4-dependent oxidative stress by Snail. miR/miRNA, microRNA; TGF, transforming growth factor; Nox4, NAPDH oxidase 4; Smad, mothers against decapentaplegic homolog.

miRNA-30e/Snail/TGF- $\beta$ /Nox4 signaling pathway to reduce oxidative stress and negative feedback regulates ROS production in AS model (Fig. 9). The results of the present study provide novel insights for miRNA in defending against oxidative stress, which miRNA-30e may represent a novel target for AS.

\section{Acknowledgements}

Not applicable.

\section{Funding}

No funding was received.

\section{Availability of data and materials}

The analyzed data sets generated during the study are available from the corresponding author on reasonable request.

\section{Authors' contributions}

YC designed the experiments. $\mathrm{MZ}$ and $\mathrm{WZ}$ performed the experiments. YC and MZ analyzed the data. MZ wrote the manuscript. All authors read and approved the final manuscript. 


\section{Ethics approval and consent to participate}

Animal experiments were approved by the Animal Care and Utilization Committee of Xiamen Cardiovascular Hospital Xiamen University.

\section{Patient consent for publication}

Not applicable.

\section{Competing interests}

The authors declare that they have no competing interests.

\section{References}

1. Wang SK, Green LA, Drucker NA, Motaganahalli RL, Fajardo A and Murphy MP: Rationale and design of the clinical and histologic analysis of mesenchymal stromal cells in AmPutations (CHAMP) trial investigating the therapeutic mechanism of mesenchymal stromal cells in the treatment of critical limb ischemia. J Vasc Surg 68: 176-181.e1, 2018.

2. Delgado-Lista J, Perez-Martinez P, Garcia-Rios A, AlcalaDiaz JF, Perez-Caballero AI, Gomez-Delgado F, Fuentes F, Quintana-Navarro G, Lopez-Segura F, Ortiz-Morales AM, et al: CORonary Diet Intervention with Olive oil and cardiovascular PREVention study (the CORDIOPREV study): Rationale, methods, and baseline characteristics: A clinical trial comparing the efficacy of a Mediterranean diet rich in olive oil versus a low-fat diet on cardiovascular disease in coronary patients. Am Heart J 177: 42-50, 2016.

3. Nicholls SJ, Puri R, Wolski K, Ballantyne CM, Barter PJ, Brewer HB, Kastelein JJ, Hu B, Uno K, Kataoka Y, et al: Effect of the BET protein inhibitor, RVX-208, on progression of Coronary Atherosclerosis: Results of the Phase 2b, Randomized, Double-Blind, Multicenter, ASSURE Trial. Am J Cardiovasc Drugs 16: 55-65, 2016.

4. Wei Y, Zhu M and Schober A: Macrophage MicroRNAs as therapeutic targets for atherosclerosis, metabolic syndrome, and cancer. Int J Mol Sci 19: pii: E1756, 2018

5. Zhang X, Shi H, Wang Y,Hu J, Sun Z and Xu S: Down-regulation of hsa-miR-148b inhibits vascular smooth muscle cells proliferation and migration by directly targeting HSP90 in atherosclerosis. Am J Transl Res 9: 629-637, 2017.

6. Stanek A, Cholewka A, Wielkoszynski T, Romuk E and Sieroń A: Whole-body cryotherapy decreases the levels of inflammatory, oxidative stress, and atherosclerosis plaque markers in male patients with active-phase ankylosing spondylitis in the absence of classical cardiovascular risk factors. Mediators Inflamm 2018: 8592532,2018

7. Ahotupa M: Oxidized lipoprotein lipids and atherosclerosis. Free Radic Res 51: 439-447, 2017.

8. Wang Y, Wang W, Wang N, Tall AR and Tabas I: Mitochondrial oxidative stress promotes atherosclerosis and neutrophil extracellular traps in aged mice. Arterioscler Thromb Vasc Biol 37 e99-e107, 2017.

9. Hassan MO and Duarte R, Dix-Peek T, Dickens C, Naidoo S, Vachiat A, Grinter S, Manga P and Naicker S: Transforming growth factor- $\beta$ protects against inflammation-related atherosclerosis in South African CKD Patients. Int J Nephrol 2018: 8702372, 2018.
10. Terada K, Yamada H,Kikai M, Wakana N, Yamamoto K, Wada N, Motoyama S, Saburi M, Sugimoto T, Irie D, et al: Transplantation of periaortic adipose tissue inhibits atherosclerosis in apoE $\mathrm{E}^{-/}$ mice by evoking TGF- $\beta 1$-mediated anti-inflammatory response in transplanted graft. Biochem Biophys Res Commun 501: 145-151, 2018.

11. Xu H, Wang Z, Sun Z, Ni Y and Zheng L: GATA4 protects against hyperglycemiainduced endothelial dysfunction by regulating NOX4 transcription. Mol Med Rep 17: 1485-1492, 2018.

12. Hu P, Wu X, Khandelwal AR, Yu W, Xu Z, Chen L, Yang J, Weisbrod RM, Lee KSS, Seta F, et al: Endothelial Nox4-based NADPH oxidase regulates atherosclerosis via soluble epoxide hydrolase. Biochim Biophys Acta Mol Basis Dis 1863: 1382-1391, 2017.

13. Shen D, Tian L, Shen T, Sun H and Liu P: Alpha-lipoic acid protects human aortic endothelial cells against $\mathrm{H} 2 \mathrm{O} 2$-induced injury and inhibits atherosclerosis in ovariectomized low density lipoprotein receptor knock-out mice. Cell Physiol Biochem 47: 2261-2277, 2018

14. Lai L, Chen J, Wang N, Zhu G, Duan X and Ling F: MiRNA-30e mediated cardioprotection of ACE2 in rats with Doxorubicin-induced heart failure through inhibiting cardiomyocytes autophagy. Life Sci 169: 69-75, 2017.

15. Livak KJ and Schmittgen TD: Analysis of relative gene expression data using real-time quantitative PCR and the 2(-Delta Delta C(T)) method. Methods 25: 402-408, 2001.

16. Misialek JR, Bekwelem W, Chen LY, Loehr LR, Agarwal SK, Soliman EZ, Norby FL and Alonso A: Association of white blood cell count and differential with the incidence of atrial fibrillation: The atherosclerosis risk in communities (ARIC) Study. PLoS One 10: e0136219, 2015.

17. Karunakaran D and Rayner KJ: Macrophage miRNAs in atherosclerosis. Biochim Biophys Acta 1861: 2087-2093, 2016.

18. Ma S, Tian XY, Zhang Y, Mu C, Shen H, Bismuth J, Pownall HJ, Huang Y and Wong WT: E-selectin-targeting delivery of microRNAs by microparticles ameliorates endothelial inflammation and atherosclerosis. Sci Rep 6: 22910, 2016.

19. Meng X, Su W, Tao X, Sun M, Ying R, Wei W and Wang B: Oxidation prevents HMGB1 inhibition on PDGF-induced differentiation of multipotent vascular stem cells to smooth muscle cells: A possible mechanism linking oxidative stress to atherosclerosis. Biomed Res Int 2018: 4019814, 2018.

20. Jin X, Yu MS, Huang Y, Xiang Z and Chen YP: MiR-30e-UCP2 pathway regulates alcoholic hepatitis progress by influencing ATP and hydrogen peroxide expression. Oncotarget 8: 64294-64302, 2017.

21. Gopoju R, Panangipalli S and Kotamraju S: Metformin treatment prevents SREBP2-mediated cholesterol uptake and improves lipid homeostasis during oxidative stress-induced atherosclerosis. Free Radic Biol Med 118: 85-97, 2018.

22. Zhang W, Chang H, Zhang $\mathrm{H}$ and Zhang L: MiR-30e attenuates isoproterenol-induced cardiac fibrosis through suppressing Snai1/TGF- $\beta$ Signaling. J Cardiovasc Pharmacol 70: 362-368, 2017.

23. Zhao W, Feng H, Guo S, Han Y and Chen X: Danshenol A inhibits TNF- $\alpha$-induced expression of intercellular adhesion molecule-1 (ICAM-1) mediated by NOX4 in endothelial cells. Sci Rep 7: 12953, 2017.

This work is licensed under a Creative Commons Attribution-NonCommercial-NoDerivatives 4.0 International (CC BY-NC-ND 4.0) License. 